\title{
The widths of vacuum-ultraviolet spectral lines in the equatorial solar corona observed with CDS and SUMER
}

\author{
K. Wilhelm ${ }^{1}$, A. Fludra ${ }^{2}$, L. Teriaca ${ }^{1}$, R. A. Harrison ${ }^{2}$, B. N. Dwivedi ${ }^{1,3}$, and C. D. Pike ${ }^{2}$ \\ 1 Max-Planck-Institut für Sonnensystemforschung, Max-Planck-Str. 2, 37191 Katlenburg-Lindau, Germany \\ e-mail: wilhelm@mps .mpg. de \\ 2 Space Science and Technology Dept., Rutherford Appleton Laboratory, Chilton, Didcot, Oxfordshire, OX11 0QX, UK \\ 3 Department of Applied Physics, Banaras Hindu University, Varanasi-221005, India
}

Received 1 December 2004 / Accepted 3 February 2005

\begin{abstract}
Observations of the solar equatorial corona between heights of $36 \mathrm{Mm}$ and $184 \mathrm{Mm}$ above the limb obtained by the SOHO spectrometers CDS and SUMER in December 2003 are presented and discussed with special emphasis on the widths of the spectral lines $\mathrm{MgX}$ at $62.50 \mathrm{~nm}, \mathrm{Al} \mathrm{XI}$ at $55.00 \mathrm{~nm}$ and $56.82 \mathrm{~nm}, \mathrm{CaX}$ at $55.78 \mathrm{~nm}$, and Si XI at $58.09 \mathrm{~nm}$. SUMER observed, in addition, the lines Mg X 60.98 nm, Ca X 57.40 nm, Fe XII 124.20 nm, Fe XVII 115.31 nm, and Ca XIII 113.37 nm. The Si XII $52.11 \mathrm{~nm}$ line was only observed by CDS. A different behaviour of the line width of $\mathrm{Mg} \mathrm{X} 62.50 \mathrm{~nm}$ as a function of height above the limb had been found in studies carried out independently with both instruments at different times. It is the aim of this joint investigation to (a) study instrumental effects on line-width results; and (b) provide a thorough analysis of line profiles with altitude for the new campaign.
\end{abstract}

Key words. Sun: corona - Sun: UV radiation - line: profiles - instrumentation: spectrographs

\section{Introduction}

Spectral lines emitted by ions of the hot corona and their characteristics provide important information on the physical conditions and processes in the magnetized plasma of the solar atmosphere. One of the critical parameters is the line width, which is influenced, in addition to the narrow intrinsic atomic width, by the Doppler broadening due to the ion temperature, turbulence and unresolved wave activity ${ }^{1}$. Before physical inferences can be drawn, the observational data must be determined as accurately and precisely as possible. In this context, the instrumental performances play a critical rôle. The previous measurements of the widths of the $\mathrm{Mg} \mathrm{X}$ lines in the low corona under quiet solar conditions with either the Coronal Diagnostic Spectrometer (CDS) or the Solar Ultraviolet Measurements of Emitted Radiation (SUMER) spectrometer had been performed at different times in independent studies (Doschek \& Feldman 2000; Harrison et al. 2002; O'Shea et al. 2003; Wilhelm et al. 2004). Whilst there have been reports of emission-line broadening with altitude, using SUMER, the CDS observations presented by Harrison et al. (2002) appeared to show emission-line narrowing. This apparent discrepancy could be due to different solar target regions, or, indeed, to instrumental effects.

The relevant design parameters of the spectrometers will be discussed in Sect. 2. Here it should be noted that CDS and

${ }^{1}$ For a line profile of Gaussian shape, the Doppler width, $\Delta \lambda_{\mathrm{D}}$, is related to the full width at half maximum, $\Delta \lambda_{\mathrm{FWHM}}$, through $\Delta \lambda_{\mathrm{D}}=\Delta \lambda_{\mathrm{FWHM}} /(2 \sqrt{\ln 2})$.
SUMER have been conceived with different and complementary scientific goals in mind: CDS aims at covering simultaneously many spectral lines emitted over a wide range of temperatures in order to determine the temperature structure of the solar atmosphere in great detail, whereas SUMER features high spectral resolution to study the dynamics of the solar plasma with the help of the Doppler effect. Yet, in some areas both instruments have overlapping capabilities, and a comparison of joint observations can be used to verify certain measurements. Line-width observations are among those. However, the different instrumental spectral transfer functions preclude a direct comparison of the absolute line widths. What can be expected is to find consistent trends of the spatial or temporal line-width variations.

\section{Instrumentation}

The Solar and Heliospheric Observatory (SOHO) (Domingo et al. 1995) has a payload consisting of twelve experiments among which are the vacuum-ultraviolet spectrometers CDS and SUMER capable of recording spectra of the solar disk and the low corona over the combined wavelength range from $15 \mathrm{~nm}$ to $161 \mathrm{~nm}$. SOHO also carries the Extremeultraviolet Imaging Telescope (EIT; Delaboudinière et al. 1995), which is able to provide context images in four narrow spectral bands centred on $17.1 \mathrm{~nm}$ (Fe IX-X), $19.5 \mathrm{~nm}$ (Fe XII), $28.4 \mathrm{~nm}$ (Fe XV), and $30.4 \mathrm{~nm}$ (He II), where the dominant spectra are given in parentheses. 


\subsection{The CDS normal-incidence spectrometer}

The characteristics of CDS are described by Harrison et al. (1995, 1997). CDS consists of a grazing-incidence Wolter-Schwartzchild Type-2 telescope feeding a normalincidence spectrometer (NIS) and a grazing-incidence spectrometer (GIS). NIS disperses the image of the slit onto a two-dimensional detector giving spectral images in two wavelength ranges (NIS 1: $30.8 \mathrm{~nm}$ to $38.1 \mathrm{~nm}$ and NIS 2: $51.3 \mathrm{~nm}$ to $63.3 \mathrm{~nm}$ ) with spectral resolution elements of $7 \mathrm{pm}$ (NIS 1) and $12 \mathrm{pm}$ (NIS 2). Either the full spectrum or selected spectral windows are transmitted to the ground. Spectral lines observed in the first order of diffraction with NIS 2 can be observed by SUMER in the second order allowing a comparison between the measurements obtained with the two instruments. Thus, only lines observable in the first order with NIS 2 will be discussed in the present paper.

\subsection{The SUMER spectrometer}

The SUMER instrument and its performance are outlined by Wilhelm et al. (1995, 1997) and Lemaire et al. (1997). The observations in this study have been obtained with detector A. Since the previous $\mathrm{Mg}$ X line-width measurements were performed with detector B (Doschek \& Feldman 2000; Wilhelm et al. 2004), this might be considered to be a disadvantage, but the spectral performance of detector $\mathrm{A}$ is superior to that of detector B and also better known, which speaks in favour of the present choice. The SUMER line-width correction ${ }^{2}$ performs a de-convolution using lookup tables. The values in the table are synthesized through a convolution process from the actual Doppler line width, the instrumental line width of $\Delta \lambda_{\mathrm{I}}=5.7 \mathrm{pm}$ with detector A, the narrow slit of $0.3^{\prime \prime}$, and the selected slit width (in the shape of a boxcar function). The correction transforms the measured line width, $\Delta \lambda_{\mathrm{FWHM}}$, into the corrected Doppler width, $\Delta \lambda_{\mathrm{D}}{ }^{*}$. A consistency check of the corrections for slits Nos. 1 and 2 will be made in Sect. 4.2. The spectral resolution elements are $\approx 4.4 \mathrm{pm}$ near $120 \mathrm{~nm}$ in the first order and $\approx 2.2 \mathrm{pm}$ in the second order near $60 \mathrm{~nm}$.

\section{Observations}

In order to resolve the apparent discrepancies mentioned in Sect. 1, a joint CDS/SUMER observational sequence was proposed at the SOHO 13 meeting on Mallorca in October 2003, and successfully executed during the SOHO/MEDOC campaign in November and December 2003. It was important to coordinate the measurements in space and time as much as possible, and to look for a quiet equatorial corona. Such conditions were present above a small prominence at the east limb on 4 December 2003. We felt that the study of other spectral lines besides $\mathrm{MgX}$ might be helpful. The line selection thus includes as many strong coronal lines as possible which could be observed by the NIS-2 channel in the first order and with three spectral window settings of SUMER in both orders. Most of the lines considered and listed in

\footnotetext{
${ }^{2}$ Function con_width_funct_3.pro in the SolarSoft Library.
}

Table 1. Coronal emission lines observed by CDS and/or SUMER with their formation temperatures in a plasma in ionization equilibrium.

\begin{tabular}{|c|c|c|}
\hline $\begin{array}{l}\text { Spectral } \\
\text { line }\end{array}$ & $\begin{array}{c}\text { Wavelength }^{a} \\
\lambda / \mathrm{nm}\end{array}$ & $\begin{array}{c}\text { Formation temperature }^{b} \\
T_{\mathrm{F}} / \mathrm{K} \\
\end{array}$ \\
\hline $\mathrm{CaX}$ & 55.78 & $0.71 \times 10^{6}$ \\
\hline $\mathrm{CaX}$ & $57.40^{c}$ & $0.71 \times 10^{6}$ \\
\hline $\operatorname{Mg} X$ & $60.98^{c}$ & $1.12 \times 10^{6}$ \\
\hline $\operatorname{Mg} X$ & 62.50 & $1.12 \times 10^{6}$ \\
\hline Fe XII & $124.20^{c, d}$ & $1.35 \times 10^{6}$ \\
\hline $\mathrm{Al}$ XI & 55.00 & $1.44 \times 10^{6}$ \\
\hline $\mathrm{Al}$ XI & 56.82 & $1.44 \times 10^{6}$ \\
\hline Si XI & 58.09 & $1.58 \times 10^{6}$ \\
\hline Si XII & $52.11^{e}$ & $1.86 \times 10^{6}$ \\
\hline Ca XIII & $113.37^{c, d}$ & $2.57 \times 10^{6}$ \\
\hline Fe XVII & $115.31^{c, d}$ & $3.54 \times 10^{6}$ \\
\hline
\end{tabular}

${ }^{a}$ Wavelengths rounded to $0.01 \mathrm{~nm}$.

${ }^{b}$ Calculated from ionic-fraction data of Mazzotta et al. (1998).

${ }^{c}$ Only observed by SUMER.

${ }^{d}$ Recorded by SUMER in the first order.

${ }^{e}$ Only observed by CDS.

Table 1 are allowed transitions between the first excited configuration and the ground state in lithium- or sodium-like ions $\left(\mathrm{Mg}^{9+}, \mathrm{Al}^{10+}\right.$ and $\mathrm{Si}^{11+}: 2 \mathrm{~s}^{2} \mathrm{~S}_{1 / 2}-2 \mathrm{p}^{2} \mathrm{P}_{1 / 2,3 / 2} ; \mathrm{Ca}^{9+}$ : $\left.3 \mathrm{~s}^{2} \mathrm{~S}_{1 / 2}-3 \mathrm{p}^{2} \mathrm{P}_{1 / 2,3 / 2}\right)$. Fe XII at $124.2 \mathrm{~nm}\left(3 \mathrm{p}^{3}{ }^{4} \mathrm{P}_{3 / 2}-3 \mathrm{p}^{3}{ }^{2} \mathrm{P}_{3 / 2}\right)$ is a forbidden line, and $\mathrm{SiXI}\left(2 \mathrm{~s}{ }^{1} \mathrm{~S}_{0}-2 \mathrm{~s} 2 \mathrm{p}{ }^{3} \mathrm{P}_{1}\right)$ is an inter-combination line. The Ca XIII $\left(2 \mathrm{~s}^{2} 2 \mathrm{p}^{4}{ }^{3} \mathrm{P}_{2}-2 \mathrm{~s}^{2} 2 \mathrm{p}^{4}{ }^{1} \mathrm{D}_{2}\right)$ and Fe XVII $\left(2 \mathrm{p}^{5} 3 \mathrm{~s}^{3} \mathrm{P}_{1}-2 \mathrm{p}^{5} 3 \mathrm{~s}^{3} \mathrm{P}_{0}\right)$ lines are also listed as they are seen in the hottest parts of the corona in the west. The $\operatorname{MgX} 62.50 \mathrm{~nm}$ line is blended by the line Si X $\left(2 s^{2} 2 p{ }^{2} P_{3 / 2}-2 s 2 p^{2}{ }^{4} P_{5 / 2}\right)$ at $62.47 \mathrm{~nm}$. Other chromospheric and transition-region lines near the $\mathrm{MgX}$ doublet and their potential influence on the line-width measurements are discussed by Wilhelm et al. (2004) with the result that a direct effect could only be expected up to $40^{\prime \prime}$ above the limb. However, the widths could be affected indirectly by scattered radiation within the SUMER instrument.

The contribution functions in Fig. 1 of the ionic lines with wavelengths near the $\mathrm{MgX}$ doublet, and of the other lines treated in this study, reflect their temperature dependence. The chromospheric and transition-region lines are well separated from the coronal lines at a temperature of about $5 \times 10^{5} \mathrm{~K}$, and will not blend the $\mathrm{Mg} \mathrm{X}$ lines in the corona. The Si X blend of the $62.50 \mathrm{~nm}$ line has a contribution function that is similar to the one of $\mathrm{Mg} \mathrm{X}$, except for the high-temperature tail of the lithium-like magnesium ion, but, as an inter-combination line, is slightly sensitive to the electron density, $n_{\mathrm{e}}$.

The joint measurements were performed near the east limb. The CDS observations are given in Table 2. The corresponding SUMER sequences in Table 3 were executed from 16:32 to 18:53 UTC. The solar radius, $R_{\odot}$, seen from SOHO was 983.3" at that time, and thus $1^{\prime \prime}$ in angular extent corresponds to $708 \mathrm{~km}$ at the Sun. The pointing locations of the spectrometers are sketched in Figs. $2 \mathrm{a}$ and $2 \mathrm{~b}$ superimposed on He II and Fe XII solar images taken by EIT. In the 


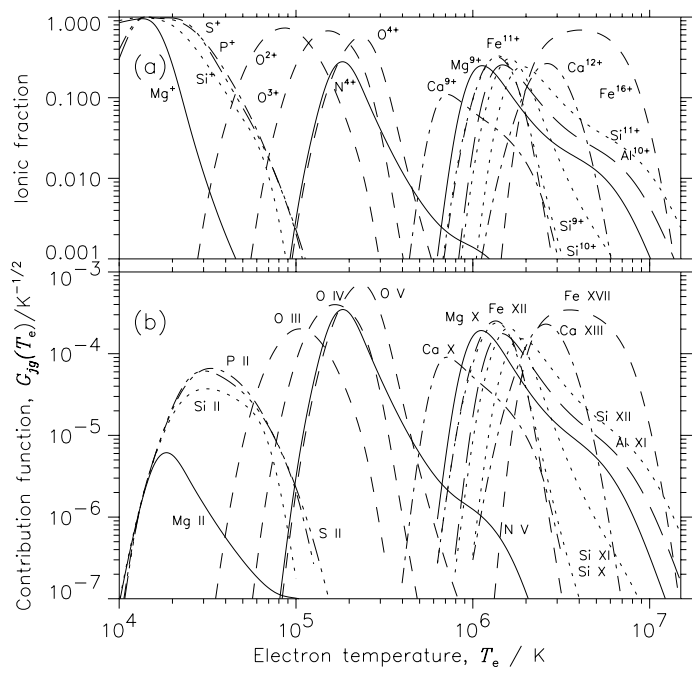

Fig. 1. a) Ionic fractions of various ions as a function of the electron temperature of a plasma in ionization equilibrium (data taken from Mazzotta et al. 1998). b) The corresponding contribution functions. The electron temperatures of their maxima are defined as formation temperatures, $T_{\mathrm{F}}$. The abundances of the various elements have not been taken into account, and thus the contribution functions can only be quantitatively compared for ions of the same element.

Table 2. CDS observations of the equatorial solar corona above the east limb on 4 December 2003. In each raster, all lines were observed.

\begin{tabular}{|c|c|c|c|}
\hline $\begin{array}{c}\text { Raster } \\
\#\end{array}$ & $\begin{array}{c}\text { Start time } \\
\text { UTC }\end{array}$ & $\begin{array}{c}\text { End time } \\
\text { UTC }\end{array}$ & $\begin{array}{c}\text { Observed lines } \\
\lambda / \mathrm{nm}\end{array}$ \\
\hline 1 & $12: 42: 48$ & $14: 23: 34$ & Mg X 62.50, Si XI 58.09 \\
\hline 2 & $14: 25: 21$ & 16:06:05 & Al XI 55.00, Ca X 55.78 \\
\hline 3 & $16: 07: 52$ & $17: 48: 35$ & Al XI 56.82, Si XII 52.11 \\
\hline 4 & $17: 50: 22$ & $19: 31: 04$ & He I 58.43 , O v 62.97 \\
\hline
\end{tabular}

western corona, SUMER made additional exposures with a similar observational sequence from 13:02 to 15:06 UTC. Since the corona was very hot there, the slit positions are shown together with the Fe XII and Fe XV windows of EIT in Figs. 2c and $2 \mathrm{~d}$.

The CDS sequences consist of four identical rasters each covering in 60 exposures a $240^{\prime \prime} \times 240^{\prime \prime}$ field of view (FOV) centred on the solar equator $\left(y=0^{\prime \prime}\right)$. The rasters started at $x=-975^{\prime \prime}$ and ended at $x=-1215^{\prime \prime}$, where $x$ is the heliocentric east-west coordinate and $y$ the north-south one. They were performed by moving the $4^{\prime \prime} \times 240^{\prime \prime}$ slit eastwards in 59 steps of $4^{\prime \prime}$. The effective exposure time at each raster position was $82 \mathrm{~s}$, after which 20 spectral windows (encompassing the relevant emission lines) were telemetered to the ground. Due to telemetry constraints spectra were binned over two pixels along the slit before transmission, yielding a final spatial resolution element of $4^{\prime \prime} \times 3.4^{\prime \prime}$. The FOV is, hence, sampled in 60 slit positions (in the east-west direction) and 72 spatial positions along the slit (north-south direction).

The number of SUMER exposures was chosen to ensure an adequate counting statistics. The height, $h$, listed in Table 3 is the tangent height, i.e. the closest approach of the slit projection to the limb along the line of sight (LOS). The previous
Mg X observations analysed by Doschek \& Feldman (2000) and Wilhelm et al. (2004) had been obtained during a SOHO roll manœuvre, when the SUMER slit was in an east-west orientation. This provided a continuous coverage of all heights covered by the slit. The present pointing configuration - without a SOHO roll - can only sequentially observe discrete height ranges in the equatorial corona, but allows us to improve the counting statistics by integrating the data along the slit. The slit No. 2 was used for the bright lines and the wide slit No. 1 for the fainter ones. Exposures with both slits were taken for a check of the correction procedures at a height of $114 \mathrm{Mm}$ (cf. Sect. 4.2). As an example we show in Fig. 3a the exposure in the $\mathrm{MgX}$ spectral window taken at a slit centre position of $x=-1034^{\prime \prime}, y=0^{\prime \prime}$. The measurements at this height ( $h=36 \mathrm{Mm}$ ) were influenced by the prominence at the east limb, but at greater heights the corona was undisturbed as can be seen from Fig. 3b. Figure 3c demonstrates that the corona was very hot near the western limb. Three features point to this fact: the lack of any Si VII and Ca VIII lines, the enhancement of the hot Ca XIII line, and the appearance of the very hot Fe XVII line.

\section{Data reduction}

\section{1. $C D S$}

The CDS spectra ${ }^{3}$ were first cleaned for cosmic-ray events rejecting entirely the profiles where spikes occurred on the body of the line. Affected pixels away from the line were treated as missing pixels. After de-biasing and calibrating, spectra were interpolated to correct for several distortions, such as the rotation of the two spectral ranges, the individual spectral line tilts, and the pointing-dependent spatial shift of the NIS-1 spectrum relative to the NIS-2 spectrum. For each line, the four corrected and calibrated rasters were summed to obtain a data cube that was then integrated along the slit direction to provide a final array of 60 high signal-to-noise spectra at different heights across and above the limb. The analysis of the behaviour of the line widths and radiances versus height for all four rasters shows that no significant differences occurred during the time needed to acquire the rasters. From Fig. $2 b$ it can be seen that there were brighter regions in the north and south extremes of the CDS FOV. The integration along the slit was, hence, performed between the spatial pixels 16 and 56, which correspond to the 160 central pixels of SUMER.

CDS spectra acquired after the SOHO recovery in 1998 are characterized by broad and asymmetric line profiles that can be fitted using a template provided within the CDS software ${ }^{4}$. The template consists of a Gaussian component (with all parameters free to vary) and a wing component whose parameters are established for NIS-2 spectra. The width measured by fitting the above template to the spectra is the width of the Gaussian component, $\Delta \lambda_{\text {FWHM }}$, which can be converted into Doppler widths

\footnotetext{
3 All raw data acquired are in the public domain and can be obtained from the SOHO Archive at

http://soho.nascom.nasa.gov/data/catalogues/main.html

4 See the CDS Software Note 53 by W.T. Thompson at http://solar.bnsc.rl.ac.uk/software/notes.shtml
} 
Table 3. SUMER observations of the equatorial solar corona above the east and west limbs on 4 December 2003.

\begin{tabular}{lcccccccl}
\hline \hline Height, $h / \mathrm{Mm}$ & $36^{a}$ & 58 & 86 & 114 & 143 & 178 & Spectral window $^{b},\left(\lambda_{20}, \lambda_{21}\right) / \mathrm{nm} ;$ & lines $^{c}$ \\
\multicolumn{1}{c}{$d /^{\prime \prime}$} & 51 & 81 & 121 & 161 & 201 & 251 & & \\
\hline Slit No. $^{d}$ & 2 & 2 & 2 & 1,2 & 2 & 2 & $\# 1: 60.85,62.87 ;$ & Mg X, Fe XII \\
Exposures $^{e}$ & 1 & 1 & 1 & 2 & 1 & 2 & & \\
\hline Slit No. $^{d}$ & 1 & 1 & 1 & 1 & 1 & 1 & $\# 2: 56.40,58.44 ;$ & Ca X, Ca XIII, Al XI, Si XI, Fe XVII \\
Exposures $^{e}$ & 1 & 1 & 1 & 1 & 2 & 3 & & \\
\hline Slit No. $^{d}$ & 1 & 1 & 1 & 1 & 1 & 1 & $\# 3: 54.87,56.93 ;$ & Ca X, Ca XIII, Al XI \\
Exposures $^{e}$ & 1 & 1 & 1 & 1 & 2 & 3 & & \\
\hline
\end{tabular}

${ }^{a}$ At the lowest height, no observations were made above the west limb.

${ }^{b}$ Second-order values of $\lambda_{2}$ without attenuator sections of the detector (cf. Fig. 3).

${ }^{c}$ Some lines are contained in more than one window.

${ }^{d}$ Slit No. 1: 4" $\times 300^{\prime \prime}$; slit No. 2: 1" $\times 300^{\prime \prime}$ (slit sizes in angular width and length).

${ }^{e}$ Sampling time: $300 \mathrm{~s}$.
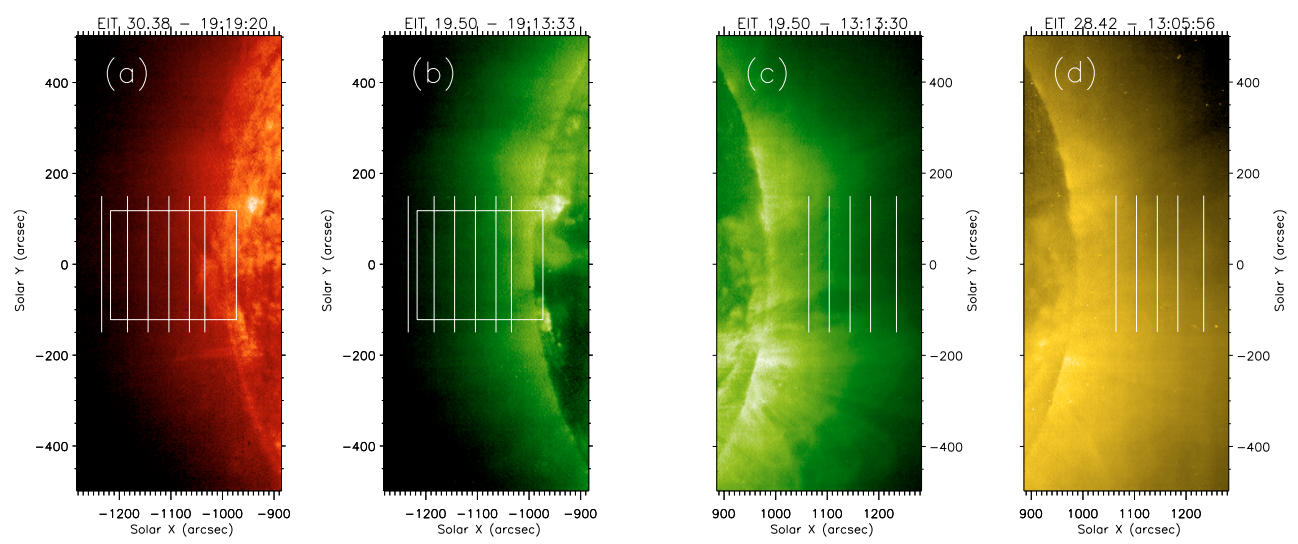

Fig. 2. Positions of the CDS FOVs (rectangles) and the SUMER slit pointing locations in relation to He II, Fe XII and Fe XV solar images of 4 December 2003 (courtesy of the EIT consortium). Joint observations were obtained in the eastern corona. At low altitudes, a prominence caused a slight disturbance there. a) He II spectral window; and b) Fe XII window near the east limb; c) Fe XII window; and d) Fe XV window near the west limb.

by applying the formula in the footnote of Sect. 1 . The profiles are largely dominated by the instrumental width preventing a measurement of the intrinsic line width. However, if the instrumental contribution is constant during the observations, or, at least, known well enough for an adequate correction, we may still detect variations in the coronal line widths. With this in mind, the behaviour of the Doppler width, $\Delta \lambda_{D}$, of the Gaussian component is investigated as a function of the limb distance.

The widths of NIS-2 lines appear to change systematically across a raster scan due to geometrical effects within the instrument. This instrumental effect is not expected to depend on the pointing of the instrument (whether on disk or off limb) or the type of observed features. To separate the instrumental variation of the line width from a physical change that may occur in different solar features, one would have to observe a homogeneous, uniformly bright area. The best available approximation to such a uniform area can be obtained by observing quiet-Sun regions near disk centre, using the CDS synoptic study. To remove any physical fluctuations of the line width of solar origin that might be present in the quiet Sun on short spatial scales, we average data from many days of synoptic observations. To ensure that the correction is valid for the time of the observation on 4 December 2003, we use synoptic data recorded during
November and December 2003. We find that the instrumental variation of the $\mathrm{Mg}$ X $62.50 \mathrm{~nm}$ line is a linear trend that introduces a relative variation of the $\mathrm{Mg} \mathrm{X}$ line width of $8 \%$ over a $240^{\prime \prime}$ wide raster, where the line width decreases from the left to the right-hand side of the raster. A correction of this trend applied to the $\mathrm{Mg}$ X data shows that the increase with height observed in Fig. 4a is largely of instrumental origin (cf. Fig. 4b). For Si XII shown in Fig. 4c, a correction could not be deduced from the data available, and thus the $\mathrm{MgX}$ correction was applied in Fig. 4d.

\subsection{SUMER}

The SUMER data ${ }^{5}$ have been reduced using the most-recent standard software in the SolarSoft Library including telemetry decompression, deadtime, geometric and local-gain corrections, as well as flat fielding and radiometric calibration. Narrow wavelength bands around the lines have been selected

\footnotetext{
5 All raw data acquired are in the public domain and can be obtained either from the SOHO Archive or from the SUMER Image Database at http://www.linmpi.mpg.de/english/projekte/sumer/FILE/ SumerEntryPage.html.
} 


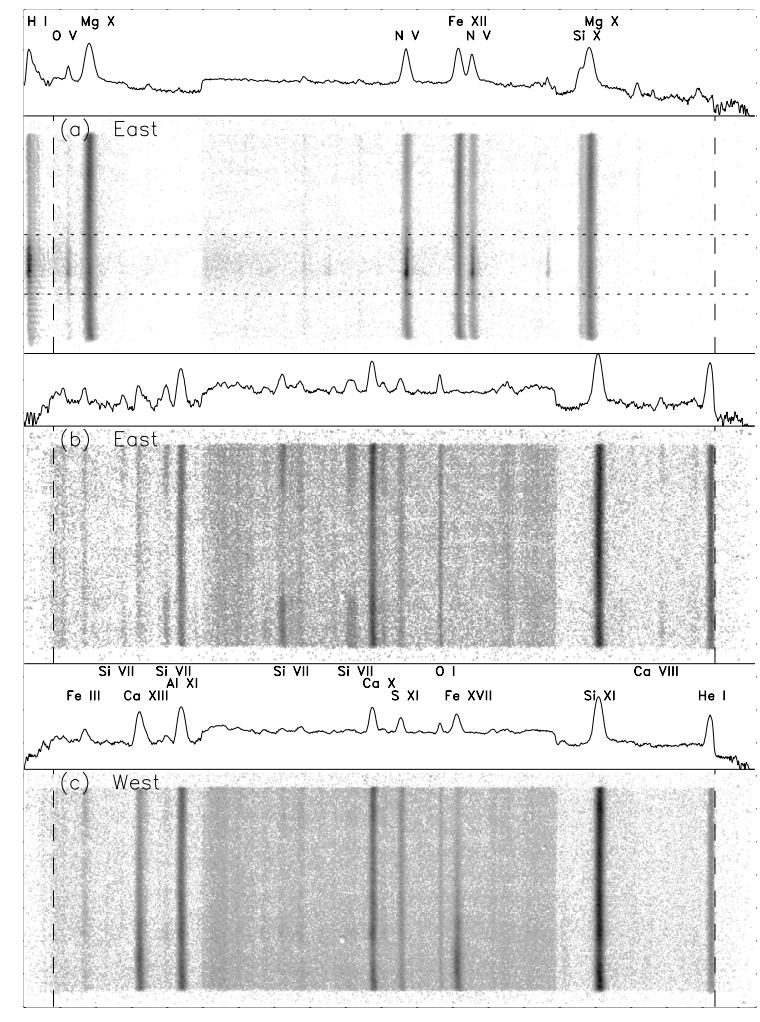

Fig. 3. Three examples of full SUMER detector exposures with 1024 spectral and 360 spatial pixels each, of which about 300 are covered by the slit images (north is up), and the corresponding spectra integrated along the slit. a) Spectral window \#1 with the $\operatorname{MgX}$ and Si X lines in the second order and the NV and Fe XII lines in the first order obtained at a angular distance of about $51^{\prime \prime}$ from the east limb of the Sun at 16:32 UTC. The extreme spectral pixels, outside of the vertical dashed lines, are covered by an attenuator of $1: 10$. The central portion with an increased continuum signal is coated with a potassium-bromide photocathode. A prominence can clearly be seen in the truncated H I Ly $\alpha$ line, the transition-region lines, and in the continuum between the dotted horizontal lines. b) The spectral window \#2 was taken at 16:56 UTC in a limb distance of $81^{\prime \prime}$. This spectrum does not show any of the prominence effects. The $\mathrm{O} I$ line is caused by instrumentally scattered radiation from the solar disk. c) The same window at the same limb distance in the west. Notice the Ca XIII and the Fe XVII lines and their spatial variations (with maxima in the south).

to produce profiles of the SUMER lines listed in Table 1 and of the N v 123.88 nm line. In Fig. 5, we show, as an example, the $\mathrm{Mg}$ X lines at different heights. In each panel, the wavelength scale was corrected for the difference between the nominal rest wavelength and the centroid of the brightest line profile. There is no significant shift of the profiles from other heights. At wavelengths longer than the centre of the $\mathrm{MgX}$ profiles, there is a plateau at a level of $\approx 0.2 \%$ of the peak radiance, which is caused by the high gain settings of the detector during the later SOHO mission times (Wilhelm et al. 2002b). This plateau was not present in the early periods of the mission (cf. Fig. $2 \mathrm{~b}$ of Wilhelm et al. 2004 with data from November 1996). The effect is, however, so small that it has no influence on the $\mathrm{Mg} \mathrm{X}$ linewidth determinations.

Previous SUMER observations of the $\mathrm{Mg} \mathrm{X}$ line widths in the equatorial region had been performed with the 4 " slit and

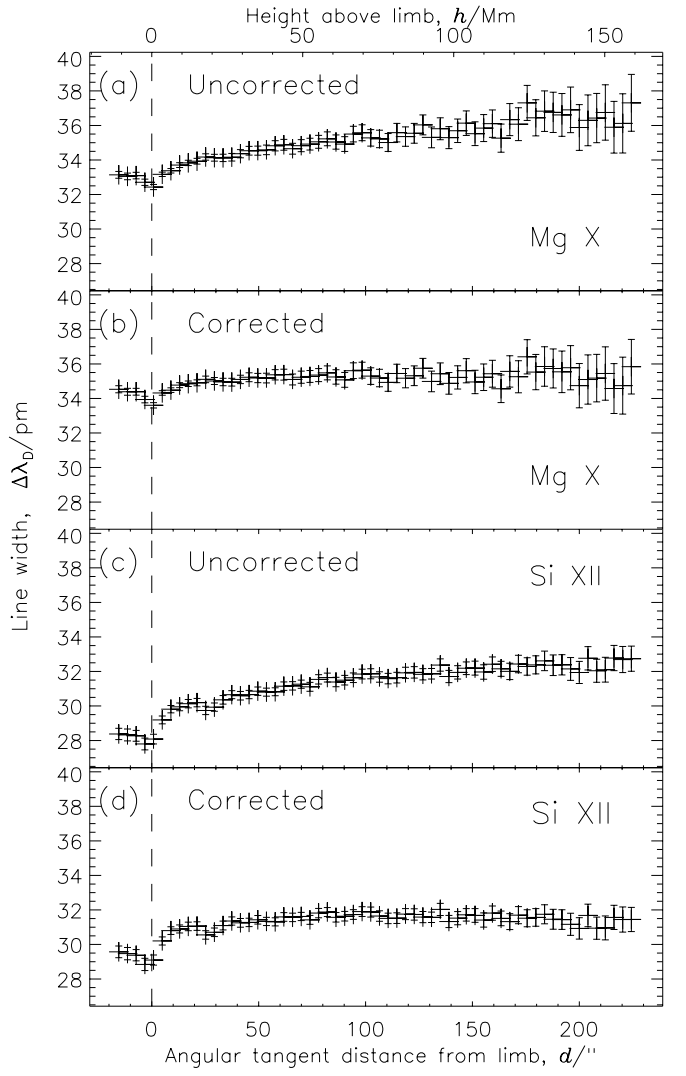

Fig. 4. Line-width variations with height as measured by CDS. a) and b) for the $\mathrm{MgX}$ line; and c) and d) for the Si XII line. The widths are plotted before and after the correction of the instrumental trend across a raster scan. In both cases the $\mathrm{Mg} \mathrm{X}$ correction was used (see text for details).

detector B. It is thus of interest to compare them with our detector A observations, and to verify that the slit-correction procedure leads to consistent results when applied to the $1^{\prime \prime}$ and $4^{\prime \prime}$ slits. Given the small line-width variations between the heights of $80^{\prime \prime}$ and $200^{\prime \prime}$, we have averaged the old Mg X measurements from $91^{\prime \prime}$ to $198^{\prime \prime}$ (cf. Table 2 of Wilhelm et al. 2004) and compared them with the new values in the range $80^{\prime \prime}$ to $210^{\prime \prime}$. We find $\Delta \lambda_{\mathrm{D}}{ }^{*}=9.26 \mathrm{pm}$ with detector $\mathrm{B}$ and the $4^{\prime \prime}$ slit and $\Delta \lambda_{\mathrm{D}}{ }^{*}=9.62 \mathrm{pm}$ with detector $\mathrm{A}$ and the $1^{\prime \prime}$ slit, consistent within the relative uncertainty estimate of $4 \%$. But, of course, the results were obtained under different conditions in the corona at different epochs. To avoid such an ambiguity, we have recorded the range $161^{\prime \prime}$ to $171^{\prime \prime}$ with both slits virtually simultaneously ( $\Delta t \approx 5 \mathrm{~min}$ ) in December 2003, and get the uncorrected and corrected widths in Table 4 . It can be concluded that the corrected line widths, $\Delta \lambda_{\mathrm{D}}{ }^{*}$, measured with both slits agree within the above uncertainty margin in both orders.

\section{Results}

The relative line width, $\Delta \lambda_{\mathrm{D}}{ }^{*} / \lambda_{0}$, is related to the ion temperature, $T_{\mathrm{i}}$, and other plasma parameters by

$c_{0} \frac{\Delta \lambda_{\mathrm{D}}{ }^{*}}{\lambda_{0}}=\sqrt{\frac{2 k_{\mathrm{B}} T_{\mathrm{i}}}{m_{\mathrm{i}}}+\xi^{2}}=\sqrt{\frac{2 k_{\mathrm{B}} T_{\mathrm{i}, \mathrm{eff}}}{m_{\mathrm{i}}}}=v_{1 / \mathrm{e}}$ 


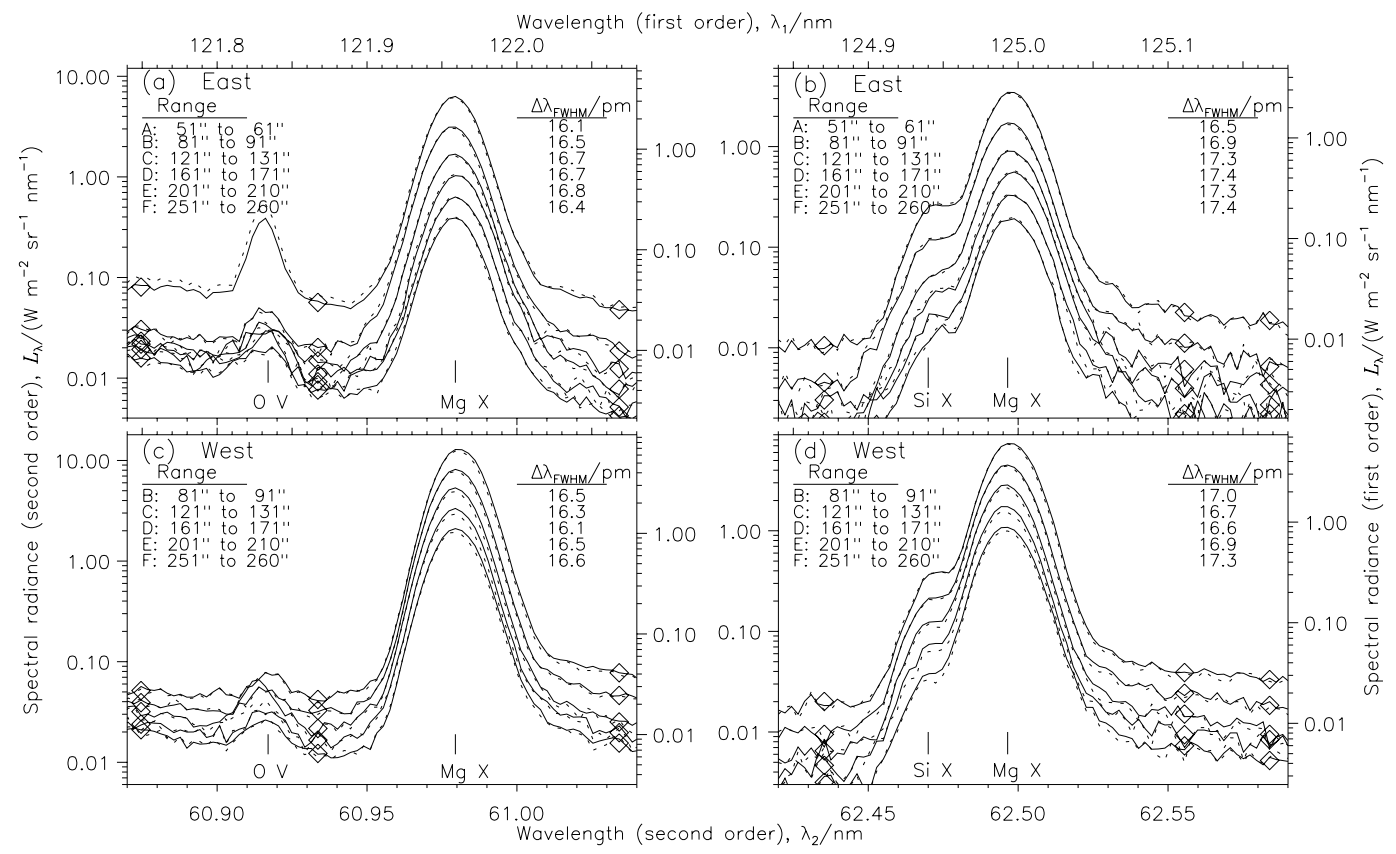

Fig. 5. The spectral radiances of the $\mathrm{Mg}$ X $60.98 \mathrm{~nm}$ and $62.50 \mathrm{~nm}$ lines observed by SUMER in the second order for heights between $36 \mathrm{Mm}$ and $184 \mathrm{Mm}$. The profiles shown in solid lines have been obtained by integrating 260 central pixels along the north-south oriented slit (cf. Fig. 2). The dotted profiles use only the 160 central pixels and are meant as a check that there are no major changes in the region covered by the slit at each position. The diamond symbols indicate the background selection for the profile fits (using a pre-conditioned multi-Gaussian approximation). The uncorrected line widths, $\Delta \lambda_{\mathrm{FWHM}}$, are indicated. a) $\mathrm{Mg} \mathrm{X} 60.98 \mathrm{~nm}$ line in the eastern corona. The $\mathrm{OV}$ at $121.83 \mathrm{~nm}$ is caused by scattered radiation from the solar disk in the instrument, except at the lowest height range, where a prominence was present. This line is seen in the first order together with the wing of the $\mathrm{HI}$ Ly $\alpha$ line as background. b) $\mathrm{Mg} \mathrm{X} 62.50 \mathrm{~nm}$ and Si X $62.47 \mathrm{~nm}$ lines in the eastern corona. They can be separated into the individual lines, resulting in a relative contribution of $\approx 8 \%$ by Si X. c), d) The same lines in the western corona.

Table 4. Uncorrected and corrected line widths at a height of $114 \mathrm{Mm}$ measured by SUMER on 4 December 2003.

\begin{tabular}{lcccc}
\hline \hline Line width & \multicolumn{2}{c}{$\Delta \lambda_{\mathrm{FWHM}} / \mathrm{pm}$} & \multicolumn{2}{c}{$\Delta \lambda_{\mathrm{D}}{ }^{*} / \mathrm{pm}$} \\
\hline Slit width & $1^{\prime \prime}$ & $4^{\prime \prime}$ & $1^{\prime \prime}$ & $4^{\prime \prime}$ \\
\hline Mg X 60.98 nm & & & & \\
$\quad$ East & 16.70 & 17.98 & 9.58 & 9.70 \\
$\quad$ West & 16.06 & 17.38 & 9.18 & 9.29 \\
Mg X 62.50 nm & & & & \\
$\quad$ East & 17.38 & 18.64 & 10.00 & 10.14 \\
$\quad$ West & 16.57 & 18.06 & 9.50 & 9.75 \\
Fe XII 124.20 nm ${ }^{a}$ & & & & \\
$\quad$ East & 27.73 & 30.11 & 15.55 & 15.34 \\
$\quad$ West & 24.99 & 28.75 & 13.78 & 14.35 \\
N v 123.88 nm ${ }^{a}$ & & & & \\
$\quad$ East & 29.61 & 32.15 & 16.76 & 16.79 \\
$\quad$ West & 28.54 & 30.10 & 16.07 & 15.33 \\
\hline
\end{tabular}

${ }^{a}$ Recorded in the first order.

(cf. Mariska 1992), where $c_{0}$ is the speed of light in vacuum, $k_{\mathrm{B}}$ the Boltzmann constant, and $m_{\mathrm{i}}$ the mass of an ion. In addition to $T_{\mathrm{i}}$, the unresolved non-thermal motions, $\xi$, contribute to the total line width and thus to the effective ion temperature, $T_{\mathrm{i}, \text { eff }}$ and the most-likely velocity along the LOS, $v_{1 / \mathrm{e}}$.

\subsection{CDS}

In Fig. 6 we have plotted the Doppler widths, $\Delta \lambda_{\mathrm{D}}$, of the Gaussian component of the $\mathrm{Mg}$ X $62.50 \mathrm{~nm}$ and Si XII $52.11 \mathrm{~nm}$ lines after correction of the raster effect. The line width is nearly constant with height above the prominence. The relative line width would be $\Delta \lambda_{\mathrm{D}} / \lambda_{0} \approx 5.6 \times 10^{-4}$, which cannot be converted into a most-likely velocity, because it contains a large instrumental contribution. The Si XI $58.09 \mathrm{~nm}$ (not shown) was also fitted. At heights smaller than $50 \mathrm{Mm}$, the fit is difficult due to the extended wings of the He I $58.43 \mathrm{~nm}$ line (strong in the prominence) in the NIS-2 channel. Above this height the radiance measured by CDS is in agreement with that obtained by SUMER, and the line width is constant with height. The weak Al XI doublet at $55.00 \mathrm{~nm}$ and $56.82 \mathrm{~nm}$ was analysed as well, but is not shown here. However, it may be of interest to note that whilst the $\mathrm{Al} \mathrm{XI} 55.00 \mathrm{~nm}$ line gave a radiance roughly consistent with that measured by SUMER, the CDS radiance of the weaker component of the doublet is much higher than expected, and totally inconsistent with the value of half the radiance of the stronger line. This may indicate blending with lines around $28.4 \mathrm{~nm}$ seen with NIS 2 in the second order. The sharp decline of the SUMER responsivity below $50 \mathrm{~nm}$ prevents these blends being present in the SUMER spectra.

\subsection{SUMER}

The relative widths of the spectral lines observed by SUMER have been plotted in Fig. 7, separately for the eastern and 


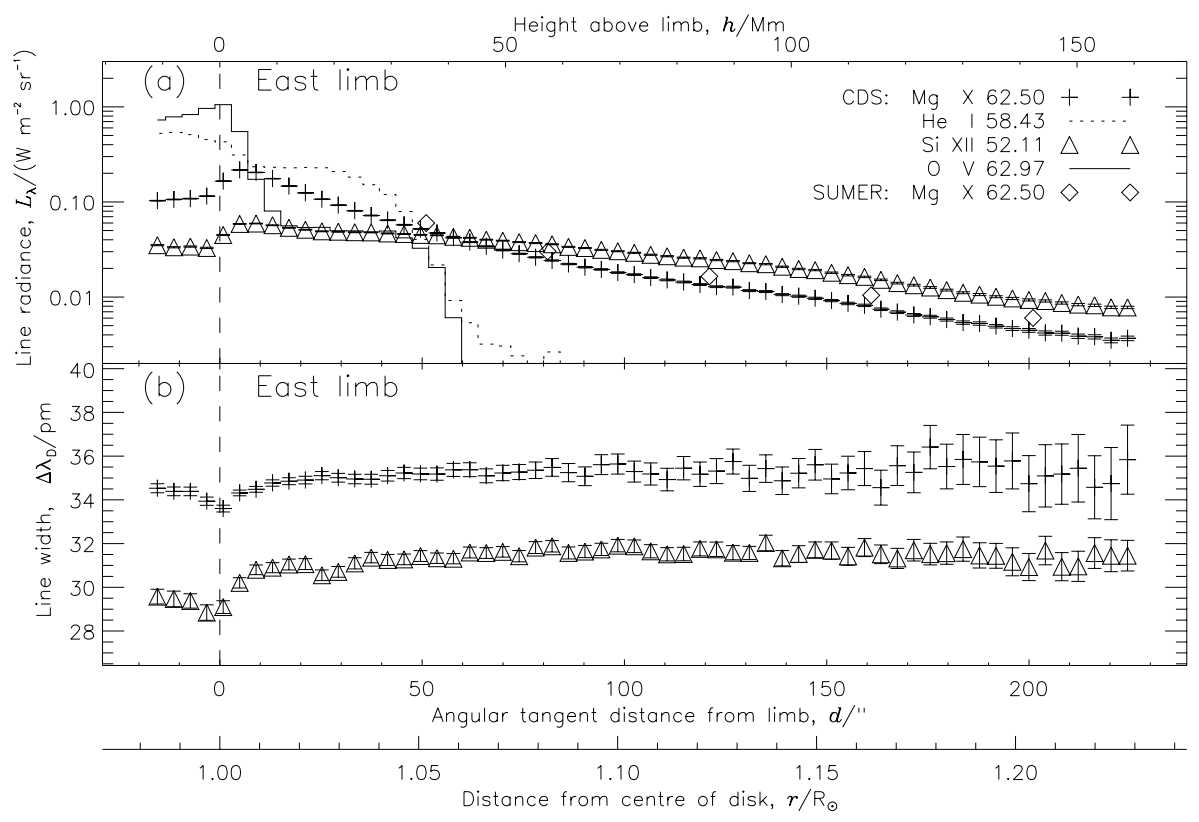

Fig. 6. Summary of the CDS radiance and line-width measurements for the coronal emission lines $\mathrm{Mg}$ X $62.50 \mathrm{~nm}$ and $\mathrm{Si}$ XII $52.11 \mathrm{~nm}$ as a function of distance from the limb. a) Radiance measurements above the east limb. For comparison, the radiances of the Mg X line measured by SUMER are indicated as well. The SUMER Mg X radiances are $\approx 20 \%$ higher with respect to those measured by CDS, a relative difference well within the combined radiometric uncertainties of both instruments (Lang et al. 2002; Wilhelm et al. 2002a), even considering the contribution of $\approx 8 \%$ by the Six $62.47 \mathrm{~nm}$ line to the CDS measurements (cf. Fig. 5). The histograms represent the radiances of the O V $62.97 \mathrm{~nm}$ and He I $58.43 \mathrm{~nm}$ lines, and show that the prominence is not important at heights greater than $50 \mathrm{Mm}$ above the limb. b) Line-width measurements are shown as Doppler widths of the Gaussian component (corrected for trends) of the line profiles of Mg X $62.50 \mathrm{~nm}$ and Si XII $52.11 \mathrm{~nm}$. The Si XII was corrected using the Mg X correction, as one specific to Si XII could not be derived. The uncertainty margins indicated in the panels represent the contributions of the counting statistics.

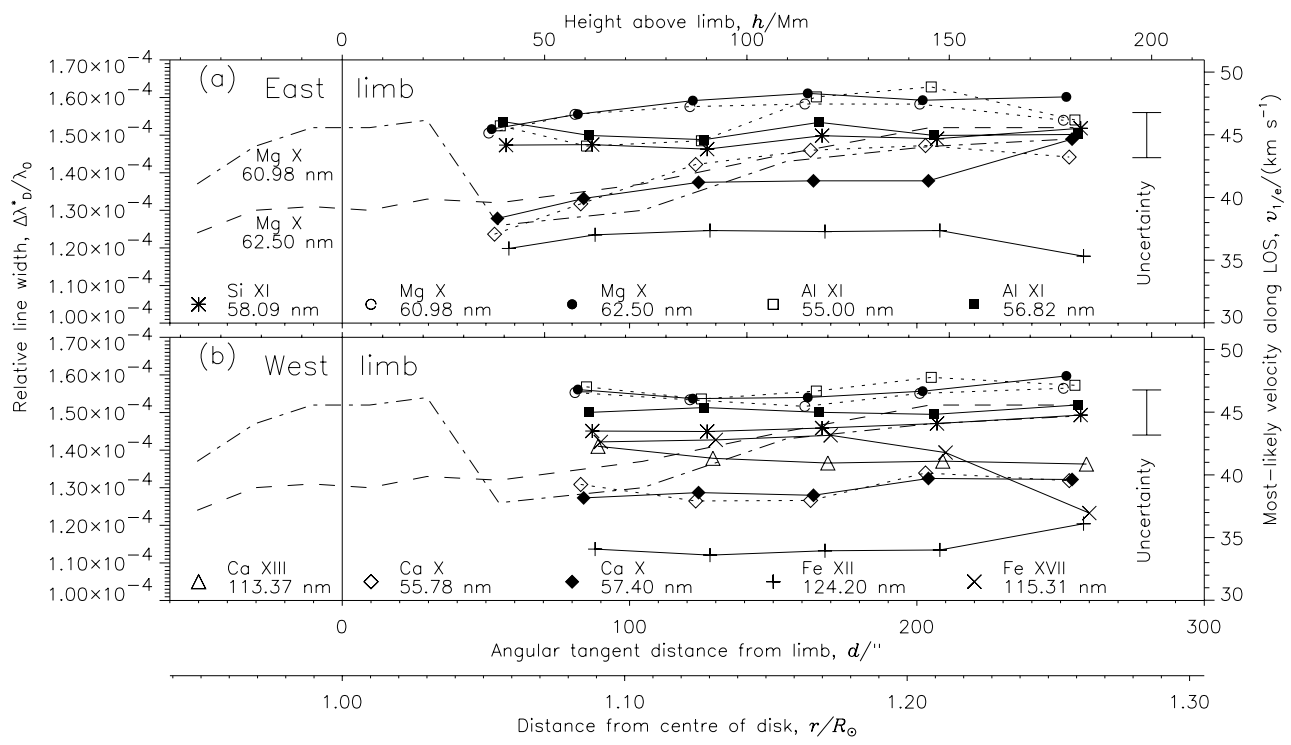

Fig. 7. Summary of the SUMER line-width measurements in terms of the relative line width, $\Delta \lambda_{\mathrm{D}}{ }^{*} / \lambda_{0}$, of the various ions, as well as their most-likely velocity along the LOS, $v_{1 / \mathrm{e}}$, as a function of distance from the limb. a) Measurements above the east limb; b) above west limb. The dashed and dash-dotted lines indicate the Mg X observations of SUMER in November 1996. The increase of the width of the $60.98 \mathrm{~nm}$ line near the limb is caused by blends of O III and O IV transition-region lines (cf. Wilhelm et al. 2004). Near the centre of the solar disk a value of $\Delta \lambda_{\mathrm{D}}{ }^{*} / \lambda_{0} \approx 1.2 \times 10^{-4}$ was observed for Mg X (Erdélyi et al. 1998; Dammasch et al. 1999). At each slit position, an altitude range of $\approx 10^{\prime \prime}$ was covered. In the interest of clarity, we spread the plot symbols of the various spectral lines over this range.

western FOVs. In cases for which more than one observation was available, mean values have been shown. The range of $v_{1 / \mathrm{e}}$ extends from $35 \mathrm{~km} \mathrm{~s}^{-1}$ to $49 \mathrm{~km} \mathrm{~s}^{-1}$ in the relatively quiet eastern corona, and from $33 \mathrm{~km} \mathrm{~s}^{-1}$ to $48 \mathrm{~km} \mathrm{~s}^{-1}$ in the active western corona. No significant differences can be noticed within the uncertainty margins. The $\mathrm{Mg} \mathrm{X}$ and $\mathrm{CaX}$ lines show 
slight increases with height in the east, but are rather constant in the west. The Fe XII line is a little narrower in the west. Of particular interest is that the lines of Ca XIII and Fe XVIII can be seen in the west, where they can be compared with the $\mathrm{CaX}$ and Fe XII lines along the same LOS.

For the purpose of a comparison with CDS, the line-width results are sufficient, but it is of great interest to attempt a separation into the thermal and non-thermal contributions. This can only be done with additional assumptions. Following Seely et al. (1997) and Tu et al. (1998), we will first assume that $\xi$ has to be equal for all ions. If we further require that all ions should have the same ion temperature, $T_{\mathrm{i}}$, we find $\xi=27 \mathrm{~km} \mathrm{~s}^{-1}$ and $T_{\mathrm{i}}=2.1 \times 10^{6} \mathrm{~K}$ for the eastern corona, but $\xi=19 \mathrm{~km} \mathrm{~s}^{-1}$ and $T_{\mathrm{i}}=2.7 \times 10^{6} \mathrm{~K}$ for the western corona (cf. Figs. $8 \mathrm{a}$ and $8 \mathrm{~b}$; horizontal dashed regression lines). In the west, we took only those lines into account that were also observed in the east. The values presented are for $h=58 \mathrm{Mm}$. At other heights, we obtained very similar results. Dolla et al. (2004) analyzed the quiet-Sun corona at $h=43 \mathrm{Mm}$ above the south pole in May 2002. Of the many lines they observed, we can compare those of the $\mathrm{Mg} \mathrm{X}$, $\mathrm{CaX}$ and Fe XII spectra with our measurements at the equator and find good agreement. The regression lines for $\xi=0$ represent the effective ion temperatures, $T_{\mathrm{i} \text {,eff }}$. It is remarkable that these temperatures are higher in the eastern corona than in the west. However, the Ca XIII and Fe XVII lines indicate much higher ion temperatures. Therefore, either our assumptions are not justified or we see different plasma regions along the LOS. The missing Si VII and Ca VIII lines speak against the presence of relative cool plasma regions in the west. For $\xi>33 \mathrm{~km} \mathrm{~s}^{-1}$ some of the Fe XII ion temperatures become negative and thus would be in a non-physical regime. Consequently, we have used $\xi=33 \mathrm{~km} \mathrm{~s}^{-1}$ as our highest non-thermal motion limit.

In Figs. $8 \mathrm{a}$ and $8 \mathrm{~b}$, the ion temperatures are plotted versus the relative atomic mass, $A_{\mathrm{i}}$, but other ordering schemes can be employed. As the gyrofrequency of an ion in a magnetic field is inversely proportional to the relative mass-over-charge ratio, $A_{\mathrm{i}} / Z_{\mathrm{i}}$, where $Z_{\mathrm{i}}$ is the ionization stage of an ion, and because the ion-cyclotron wave-particle interaction is one of the promising coronal-heating theories (e.g. Tu et al. 1998), we have displayed the ion temperatures and thermal LOS velocities versus $A_{\mathrm{i}} / Z_{\mathrm{i}}$ for $\xi=(0,19$ and 33$) \mathrm{km} \mathrm{s}^{-1}$ in Figs. $8 \mathrm{c}$ and $8 \mathrm{~d}$. If we first consider the ion temperature, we find linear relationships only if we disregard the Ca XIII and Fe XVII observations. However, we obtain good fits of the thermal velocities

$v_{\mathrm{th}}=\sqrt{\frac{2 k_{\mathrm{B}} T_{\mathrm{i}}}{m_{\mathrm{i}}}}$

along the LOS for all ions, in particular for small $\xi$.

Yet another scheme follows from the assumption that the temperature of an ion population is the same as the formation temperature of the observed emission line. The outcome is shown in Fig. 9 for all lines and heights in the western corona. We find a clear decrease from $\xi=39 \mathrm{~km} \mathrm{~s}^{-1}$ at $2 \times 10^{5} \mathrm{~K}$ to $23 \mathrm{~km} \mathrm{~s}^{-1}$ at $3.6 \times 10^{6} \mathrm{~K}$, which can be fitted by a linear regression reasonably well. The large scatter of the data points and their concentration in distinct groups suggest an additional evaluation, i.e. the calculation of mean values of $\xi$ for each
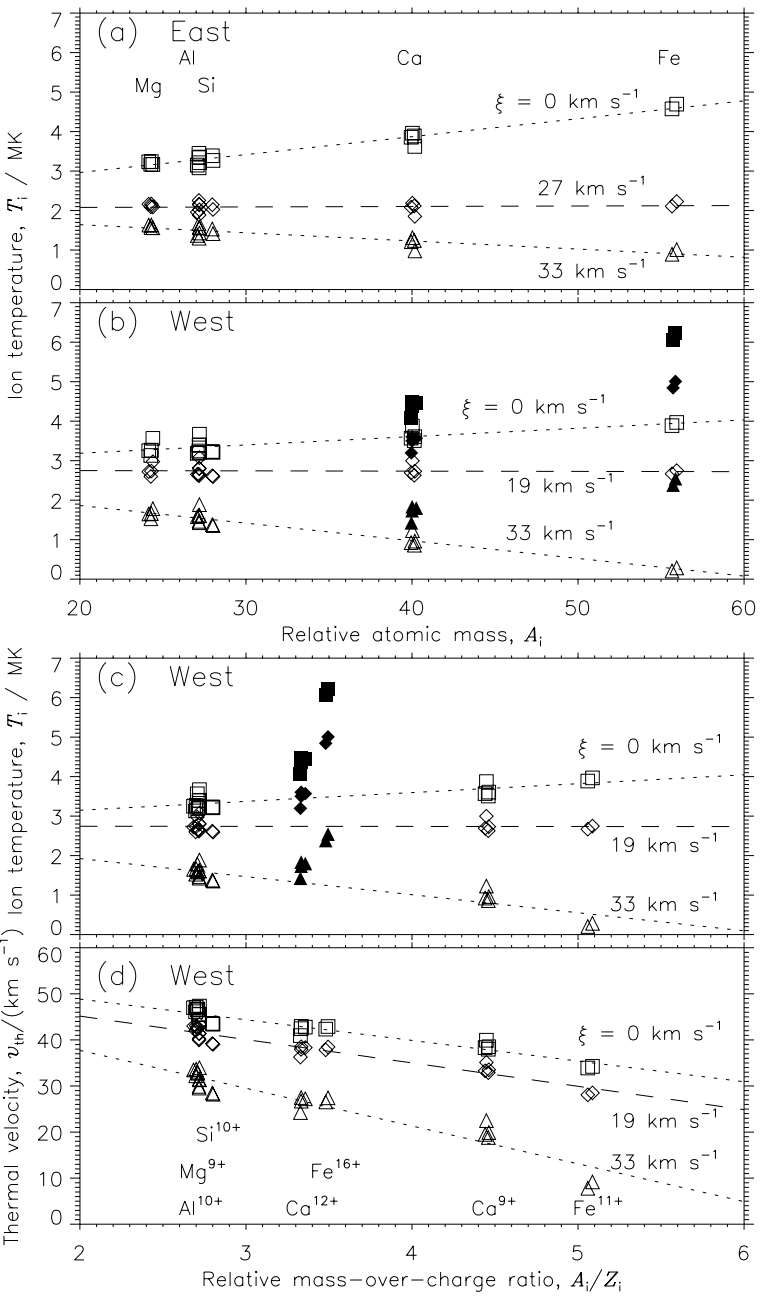

Fig. 8. The temperatures, $T_{\mathrm{i}}$, and the thermal LOS velocities, $v_{\mathrm{th}}$, of coronal ions as a function of the relative atomic mass, $A_{\mathrm{i}}$, or the relative mass-over-charge ratio, $A_{\mathrm{i}} / Z_{\mathrm{i}}$, with the non-thermal motion, $\xi$, as parameter. a) Observations from the eastern corona at $58 \mathrm{Mm}$. b) The western corona at the same height. c) The measurements presented in b) as a function of $A_{\mathrm{i}} / Z_{\mathrm{i}}$ in terms of $T_{\mathrm{i}}$ and d) as $v_{\text {th }}$ versus $A_{\mathrm{i}} / Z_{\mathrm{i}}$. Linear regression lines have been added. In panels b) and c), the hightemperature lines Ca XIII and Fe XVII are plotted separately with filled symbols and are not included in the regression calculations. Small random contributions have been added to the abscissa values to facilitate the identification of individual symbols representing the measurements.

group. We get $\bar{\xi}=38.6 \mathrm{~km} \mathrm{~s}^{-1}$ for $\mathrm{N} \mathrm{v}, 34.2 \mathrm{~km} \mathrm{~s}^{-1}$ for the coronal lines, and $25.5 \mathrm{~km} \mathrm{~s}^{-1}$ for the very hot lines.

\section{Discussion and conclusions}

In the relatively quiet equatorial corona above a small prominence, we find no or very slight increases of the line widths of coronal emission lines with altitude from measurements both with CDS and SUMER. Taking the combined uncertainty margins into account, the relative variations for $\mathrm{Mg} \mathrm{X}$ can be considered to be consistent, although the absolute widths cannot be compared given the different instrumental spectral transfer functions. The SUMER observations indicate even less line-width variations with height in the more active corona. Singh et al. (2003) found height variations of line profiles in 


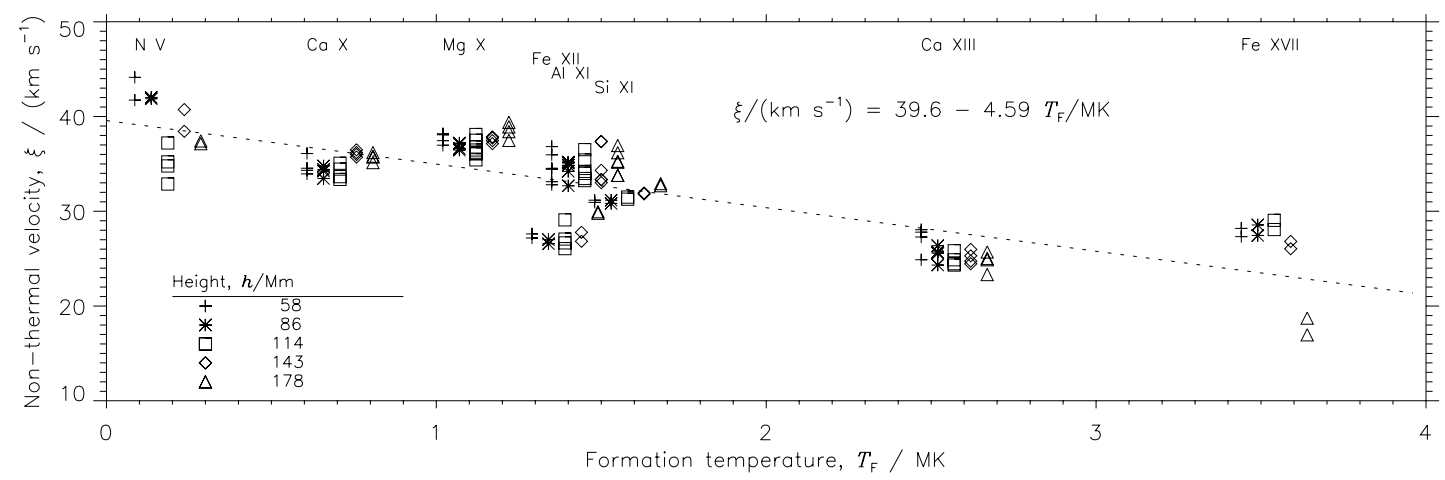

Fig. 9. Evaluation of Eq. (1) under the assumption that the ion temperatures are equal to the formation temperatures of the emission lines under consideration. The corresponding non-thermal velocities are plotted versus the formation temperature for all heights in the west. To avoid masking of the various symbols, they are spread over a range of $\pm 1 \times 10^{5} \mathrm{~K}$ in temperature for each line. The correlation coefficient of the linear regression is $r=-0.79$.

the visible light that depended on the formation temperatures of the lines. So, the solar conditions appear to have a direct influence on the line-width variations with height. Whether this dependence can account for the past apparent discrepancies cannot unambiguously be decided with the information available. More observations for different coronal activity levels are needed for this task. We have, however, achieved our main goal: CDS and SUMER relative line-width measurements do not lead to inconsistencies if the same solar region is under study.

Our attempts to use the results of SUMER for an unambiguous separation of the line widths into thermal and non-thermal contributions to the Doppler broadening were not completely successful. The best scheme for ordering the measurements is to assume no or a low constant non-thermal velocity of all ions and to plot the thermal velocity versus the relative mass-overcharge ratio (cf. Fig. 8d). This gives a linear decrease of the thermal velocities of the ions with $A_{\mathrm{i}} / Z_{\mathrm{i}}$.

Acknowledgements. CDS was built and is operated by a consortium led by the Rutherford Appleton Laboratory and including the Mullard Space Science Laboratory, the Goddard Space Flight Center, the Max-Planck-Institut für Extraterrestrische Physik, and the University of Oslo. The SUMER instrument and its operation are financed by the Deutsches Zentrum für Luft- und Raumfahrt, the Max-PlanckGesellschaft, the Centre National d'Études Spatiales, the National Aeronautics and Space Administration, and the European Space Agency's PRODEX programme (Swiss contribution). L.T. thanks Philippe Lemaire for his help in acquiring the present SUMER data set.

\section{References}

Dammasch, I. E., Hassler, D. M., Wilhelm, K., \& Curdt, W. 1999, in Proc. of 8th SOHO Workshop, Plasma Dynamics and Diagnostics in the Solar Transition Region and Corona (Noordwijk: ESA Publications Division), ESA SP-446, 263
Delaboudinière, J.-P., Artzner, G. E., Brunaud, J., et al. 1995, Sol. Phys., 162, 291

Domingo, V., Fleck, B., \& Poland, A. I. 1995, Sol. Phys., 162, 1

Dolla, L., Solomon, J., \& Lemaire, P. 2004, in Proc. of 13th SOHO Workshop, Waves, Oscillations and Small-scale Transient Events in the Solar Atmosphere: A Joint View from SOHO and TRACE (Noordwijk: ESA Publications Division), ESA SP-547, 391

Doschek, G. A., \& Feldman, U. 2000, ApJ, 529, 599

Erdélyi, R., Doyle, J. G., Perez, M. E., \& Wilhelm, K. 1998, A\&A, 337, 287

Harrison, R. A., Sawyer, E. C., Carter, M. K., et al. 1995, Sol. Phys., 162,233

Harrison, R. A., Fludra, A., Pike, C. D., et al. 1997, Sol. Phys., 170, 123

Harrison, R. A., Hood, A. W., \& Pike, C. D. 2002, A\&A, 392, 319

Lang, J., Thompson, W. T., Pike, C. D., et al. 2002, in The Radiometric Calibration of SOHO, ed. A. Pauluhn, M. C. E. Huber, \& R. V. Steiger (Noordwijk: ISSI/ESA), ISSI Scientific Report, SR-002, 105

Lemaire, P., Wilhelm, K., Curdt, W., et al. 1997, Sol. Phys., 170, 105

Mariska, J. T. 1992, The Solar Transition Region (Cambridge: Cambridge University Press)

Mazzotta, P., Mazzitelli, G., Colafrancesco, S., \& Vittorio, N. 1998, A\&AS, 133, 403

O'Shea, E., Banerjee, D., \& Poedts, S. 2003, A\&A, 400, 1065

Seely, J. F., Feldman, U., Schühle, U., et al. 1997, ApJ, 484, L87

Singh, J., Ichimoto, K., Sakurai, et al. 2003, ApJ, 585, 516

Tu, C.-Y., Marsch, W., Wilhelm, K., \& Curdt, W. 1998, ApJ, 503, 475

Wilhelm, K., Curdt, W., Marsch, E., et al. 1995, Sol. Phys., 162, 189

Wilhelm, K., Lemaire, P., Curdt, W., et al. 1997, Sol. Phys., 170, 75

Wilhelm, K., Schühle, U., Curdt, W., et al. 2002a, in The Radiometric Calibration of SOHO, ed. A. Pauluhn, M. C. E. Huber, \& R. V. Steiger (Noordwijk: ISSI/ESA), ISSI Scientific Report, SR-002, 145

Wilhelm, K., Inhester, B., \& Newmark, J. S. 2002b, A\&A, 382, 328

Wilhelm, K., Dwivedi, B. N., \& Teriaca, L. 2004, A\&A, 415, 1133 